\title{
Research On Enterprise Public and Private Cloud Service
}

\author{
Prakash Gopalakrishnan, B. Uma Maheswari
}

\begin{abstract}
In this paper, a generic comparison between the Enterprise Public and Private Cloud is analyzed based on its features and service provided. Cloud computing technology is widely adopted in today's industry where large database applications are deployed. Every organization moving towards cloud will have to consider many factors such as security, portability, database size, platform independence, multi-tenancy, customer requirements and other related resources. The detailed study focuses on essential cloud characteristics, service models, and cloud operation models such as Integrated (Hybrid), Public, Internal (Private) and Community clouds. The outcome is an effective assessment of leading two Enterprise cloud technology service providers - ownCloud ${ }^{\odot}$ and HP Public Cloud ${ }^{\circledR}$ and its implications on the organizations which used them for cloud based applications. ownCloud ${ }^{\odot}$ is the preferred open source file system for organizations to access for solutions across the world. HP Cloud offered by Hewlett-Packard (HP) provides public, private, hybrid and community cloud based services for various enterprise and other large-scale business organizations. Both the Private Cloud and Public Cloud can be enhanced to protect the data from intrusion with various security features where multiple users share the same cloud for storing and retrieving data.
\end{abstract}

Keywords- Public Cloud; Internal (Private) Cloud; Integrated (Hybrid) Cloud; Community Cloud; ownCloud ${ }^{\odot}$; HP Public Cloud ${ }^{\circledR}$.

\section{INTRODUCTION}

The term "Cloud computing" has achieved major significance in the recent developments with the wide growth of Internet technology. Cloud computing, though recently gained popularity, it was the concept introduced in the 1960s. Prof. John McCarthy, the famous Cognitive Scientist proposed that Cloud computing was restructured from the notion of Utility computing during a lecture in the MIT's centenary carnival on 1961 [1]. National Institute of Standards and Technology (NIST) fosters Cloud computing practices by setting up a framework which has to be used for the Federal Government standards. NIST defines Cloud computing as a model for setting up suitable, on-demand network access to remote servers for sharing and delivering computing resources such as Web services and other Webbased storage applications. Such remote hosted services can be transferred very fast to any other networks with minimal managerial effort and equally without any interaction of service providers [2]. NIST based cloud model supports ease of access and is defined in terms of its critical features, service models, and operational models. If the Cloud

Revised Manuscript Received on April 12, 2019.

Prakash Gopalakrishnan, Department of Computer Science and Engineering, Amrita School of Engineering, Bengaluru, Amrita Vishwa Vidyapeetham, A.P, India. (E-mail: g_prakash@blr.amrita.edu)

B. Uma Maheswari, Department of Computer Science and Engineering, Amrita School of Engineering, Bengaluru, Amrita Vishwa Vidyapeetham, A.P, India. (E-mail: b_uma@blr.amrita.edu) infrastructure is planned and designed appropriate, then the cloud operations in IT and ITES industries could able to leverage working capital costs, improve elasticity and enhance the service levels [3].

Deploying cloud increases the ultimate goal of a business organization's IT demand and supply chain to be synchronized and cost-effective. Hence it is considered to be most essential part of the today's technology. Sherif Sakr et al [4] presented the complete survey on different mechanisms and approaches for deploying exhaustive data specific applications in the cloud which are adding exponential growth to both research and industry-based communities. In their paper, decisions on different design approaches of cloud models and its exactness to provide support to certain types of applications / end-users were analysed. Apart from these research details, some generic issues and the potential challenges related to scalability, consistency, processing large-scale data economically were discussed. The features of excellent types of applications that can be operated on the cloud were emphasized.

N. Usha et al. [20], explained about the Node Deployment problem in their paper with respect to Public Cloud. They had proposed two encoding to solve the Longest Link Node Deployment Problem (LLNDP) and the Longest Path Node Deployment Problem (LPNDP). They distinguished the Private and Public Cloud in terms of applying the 5-3-2 principle and further discussed about the Cumulogic platform for development where it can be used to check the feasibility of HP's Public Cloud. The authors provided the details only for Public Cloud and its issues focused towards business community.

R. Arathi et al. [21] identified the significant issues of cloud computing as Load Balancing. In spite of various load balancing algorithms available the authors presented an enhanced HoneyBee Foraging Algorithm (HBFA) combined with the Proactive Fault Tolerance in order to prevent the unsolicited termination of the cloud access. It has been shown that out of different algorithms, the HBFA outperforms the tasks without any major loss in the execution.

\section{MATERIALS AND METHODS}

\section{A. Critical Features of Cloud}

The five essential features of cloud that enable the cloud technology to be widely adopted in most of the today's small or large scale industries are: 
On-demand self-service: Self-services of Computing includes e-mails, software applications, network administrator or server services which can be offered without involving human interaction with the service provider. Many cloud service providers such as HP Helion, AWS, Internap, IBM SoftLayer, etc are the top best companies that offer On-demand self-services. Adobe Systems, BMW, Canon, and Autodesk are some of the companies that use web services offered by AWS.

Broad network access: As time and cost associated with any communication network have decreased due to scalability and existence of many commodity products, now Cloud computing enables broad network access for multiple client platforms. These platforms include any heterogeneous thin or thick clients such as mobile phones, Personal Digital Assistants, etc.

Resource pooling: Cloud users can able to bulk access Virtual servers, storage devices, networks which are formed as pooled resources in cloud architecture. Different physical and virtual devices are assigned to the Cloud users and multiple clients using the nested and hierarchical resource pool model based on their demand and request for the service. This pooling mechanism will be considered as very economical and shall be created for live Cloud IT resources. However pooling such multiple resources could become highly complex when developed for some specific Cloud users and clients.

Rapid elasticity: In Cloud, provisioning, and deprovisioning of additional machines (virtual computers) has to be instantly done based on the service required for the users. An elastic system is supposed to detect these conditions and could able to adapt to the changes in workload without human intervention. This is a most important attribute of Cloud and its applications.

Measured service: It is clear from the past cloud strategy that the affordable nature is its inclination towards economic and fiscal policies, where any user shall pay only for what he utilized from the services provided. Any Cloud user needs a precisely configured product to be delivered. The resources attached with the product shall be storage deployment, network bandwidth performance, availability of software, CPU utilization which is measured according to the specific requirements by them. Such measuring capability will provide the exact number of resources utilized by the user. Here both the Cloud user and his Service Provider could monitor and control transparently the infrastructure provided by the Cloud.

\section{B. Cloud Service Models}

When a cloud platform is recognized, the services are organized with regard to the business models that can be distinguished based on the requirements. The commonly deployed service models are shown in Fig. 1 and are explained as follows:

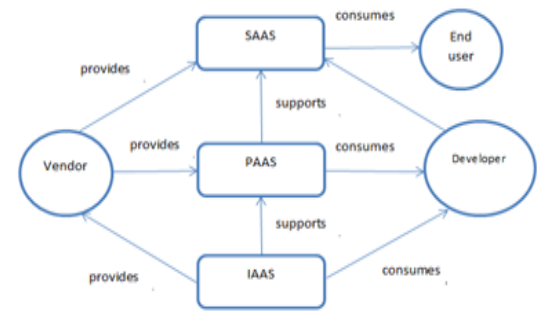

Fig. 1. Service Models

Software as a Service (SaaS) - Many licensed software applications are costly which cannot be purchased by smallscale IT industries where they expect an alternate solution. They could demand the Software service such as pay-perhour where the Service providers could provide, where they are referred as SaaS provider. Cloud technology provides Software services such that multiple users who want to access the Software share the same code-base that is centrally located. Cloud users could then make use of such software services through a thin client or program interface. SaaS users need not want to have the supporting hardware capabilities like networking devices, storage servers, etc. and only a web browser is sufficient to handle the software applications that have to be accessed. SaaS users would easily tailor the applications that fit their business needs without monitoring or controlling the common infrastructure.

Platform as a Service (PaaS) - Software Services requires a set of tools and a platform to deploy their applications for Cloud users. PaaS provide such platforms to make the software applications work effectively where SaaS is built on it. Cloud service provider allows the consumers either to deploy their self-created applications onto the cloud stack or deploy other applications obtained onto the cloud that is developed by programming languages, shared standard library routines, web-based software, tools, etc. supported by the service providers. Similar to SaaS, the Cloud users have no control over the crucial cloud operational platforms but they could manage the deployed applications and set up configuration details for the application hosting environment. Cloud users have to purchase to access the PaaS service which enables them to work on a software project using their own software in the cloud. Since there is no control for the users to manage operating systems and networks, there are certain situations that led to various constraints where the performance of applications requires customization when deployed.

Infrastructure as a Service (IaaS) - The most important on-demand service that Cloud technology provides to its users is the networks, operating systems, high-end software, and other resources that can be outsourced. Small scale IT business sectors need not want to purchase all these components as they are utilizing them at the minimum level. The entire infrastructure has been controlled by the Cloud and the resources shall be distributed to multiple users where they could manage the systems at lower cost but with the fullest utilization capabilities [17]. 


\section{Cloud Operational Models}

The entire Cloud Technology services will differ based on its requirement and deployment. There are four deployment models available which are having specific features and are defined as follows: These models normally support the services that are needed for Cloud users in definite ways.

Private Cloud - Presently, it is well known that providing free storage and resource sharing in Cloud is always a threat. Hence, many organizations are now preferred to have a separate cloud environment exclusively for their operations. A Private Cloud is supposed to provide the organization with better monitoring and controlling of the underlying cloud hardware and software infrastructure. IBM Smart Cloud Foundation, ownCloud ${ }^{\circledR}$, and Citrix cloud platforms are the examples for Private cloud providers [5]. CloudStack and OpenStack are the open source software that is used to build a Private Cloud.

Community Cloud - If a similar type of organizations wants to establish a network within their limit, then Community Cloud is the right choice where resources are shared among these groups of companies. Such organizations shall keep their capital expenses lower which shall be shared among their counterpart firms with respect to the establishment of cloud setup infrastructure. Siemens IT solutions and services data centers provide Community cloud service along with Microsoft Azure [6].

Public Cloud - An organization provided with vendor support SaaS applications are well supported with this public infrastructure which has less capital and is economically scalable. The service provider would provide all facilities such as hardware, software, network bandwidth and other resources. Cloud users have to incur only the cost associated with the utilization capacity. Hence any middlelevel organizations could deploy services using Public Cloud with modest financial expenditure when compared with the huge principal investments required for other cloud models. Google, Microsoft and Amazon Web Services are good examples for operating the Public cloud infrastructure.

Hybrid Cloud - This is the most flexible infrastructure out of all the four models as any organization could have both Public and Private Clouds based on their needs. However, the complexity increases as the clouds using this technology and its interfaces should be competent enough while permitting to transfer data from one cloud to another. Here Hybrid Cloud could make use of Private cloud inside the organization premises in a secured manner and preserve the Public Clouds for utilizing the commercial hardware and software services obtained through SaaS and PaaS. An example of the Hybrid cloud provider is VMware vCloud Air Disaster Recovery. OpenStack is the blueprint for hybrid cloud deployment.

\section{OWNCLOUD ${ }^{\odot}$ - A PRIVATE CLOUD}

Many organizations today are using cloud to synchronize files present in several locations over the Internet. Cloud vendors are providing different software for this purpose. However it is critical to maintain the security of these files in the Cloud which is always a major concern to the business enterprises. Moreover the problem lies in using the proprietary software where it finds very expensive to share the files frequently on routine work schedule.

To overcome these issues, an open source software ownCloud $^{\odot}$ is developed which offers file synchronizing and file sharing features securely. It gives the users to access files quickly through the WebDAV. It can be used to view and access the user details, sync the date and time, bookmarks across all the cloud user systems. The Server software can be downloaded and installed in the Linux Server and only minimal efforts are required without the need for special permissions. It can be extendable using a plain but authoritative API for plug-ins and other software applications [14]. On the other end, the clients shall be either desktop /mobile for data sync. Desktop clients support Windows 7, 8.x, 10 versions; Linux Distributions and Mac OS X versions whereas Mobile clients are Apple AppStore, Android Google Play and BlackBerry systems. A generic Private Cloud within an enterprise data center is depicted in Fig. 2.

Private Cloud Inside Enterprise Data Center

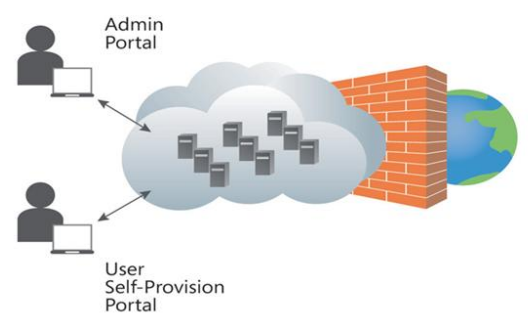

Fig. 2. Illustration of Private Cloud

OwnCloud $^{\odot}$ has the following features which makes it to be widely used as Private Cloud [15]:

i. Access, Share and Sync User Data [8]: Files, photo galleries, calendars, contacts, etc. are accessed, stored and synchronized on a server preferred by Cloud users. Stored data can be accessed and shared either public or private with other users from the user's desktop systems, mobile devices or a web browser. The files with recent versions can be obtained anytime with the desktop systems, mobile app, or web client.

ii. Versioning [8]: An important feature that should be enabled in any application for retrieving the old files, ownCloud $^{\odot}$ routinely saves the old files of the Cloud users in different versions. The decision regarding to what extent data can be saved shall be done by configuration. To revert back the changes, one's file shall be moved to the earlier version.

iii. Encryption [8]: As ownCloud ${ }^{\circledR}$ is private and used only within the organization, securing the files is significant which is achieved by encrypting them. File Firewall provides restricted access to its cloud users and administrators to follow the access rules associated with its server. The determination of file access is decided by the granularity of rules. 
iv. Drag and Drop Upload [8]: With this feature, a user need not want to install the whole ownCloud ${ }^{\odot}$ client and he could just log on to the web browser directly. Then he could drag the files from desktop systems and drop them into the preferred target folder into the web browser where the ownCloud ${ }^{\odot}$ client resides.

v. ODF Files Viewer [8]: Using this characteristic, anyone could view the open document formats and shall click on any ODF formatted document (.ods, .odp, .odt,) for reading it from the web browser without downloading.

vi. Migration, Backup and Logging [8]: Several occurrences of ownCloud ${ }^{\odot}$ installed with main and backup support could simply move ownCloud ${ }^{\odot}$ user accounts among ownCloud ${ }^{\odot}$ occurrences and shall comprise a backup set when needed. ownCloud ${ }^{\odot}$ could also be used to write in system log files in addition to the existing ownCloud ${ }^{\circledR} \log$ file which helps in keeping track of the changes and access.

vii. Tasks, Calendars and Application Store [8]: With the Tasks features enabled in the application, one could straightforwardly sync one's to-do lists with the ownCloud $^{\circledR}$ occurrence. One can share important calendar and important events by enabling the Calendar feature. Any ownCloud ${ }^{\odot}$ users shall open the Calendar and by selecting the share option shall prefer the users or their groups for whom it need to be shared. Enabling a new application in settings, one can add existing applications to ownCloud ${ }^{\odot}$, which will be downloaded automatically in the ownCloud ${ }^{\circledR}$ instance.

viii. File Notifications and Galleries [8]: The File Notification feature once given will make the files and its corresponding documents such as movies, video clips, and others to be shared quickly and easily. One can specify the ownCloud ${ }^{\odot}$ photo directories in order to have control, also sort order, share galleries with any email address by choosing, and control whether others can share those photos with anyone else.

ix. Jive ${ }^{\circledR}$ Collaboration [8]: Jive, a leading enterprise social collaboration and communication solutions is now configured for ownCloud $^{\odot}$ as a data storage center. That is the files stored in Jive could be seen in the folders of ownCloud ${ }^{\odot}$. Here Jive is a system and ownCloud $^{\odot}$ would act as a proxy that provides end to end file access on the desks of cloud users on the move.

x. LDAP/Active Directory [8]: ownCloud $^{\odot}$ enables administrators to handle users and their groups from Lightweight Directory Access Protocol or Active Directory instance.

xi. User Controls and Alerts: ownCloud ${ }^{\circledR}$ v6.0 users have new controls that improve productivity and usability. An Undelete control allows deleted files to be recovered from the trash bin. A conflict handling dialog warns users who are uploading files that already exist; and the new Activity Stream feature allows users to see all file and sharing activities associated with their files.

xii. Sharing Notifications: An optional feature in ownCloud $^{\circledR}$ v6.0 is that emails can be sent to users and groups when files or/and folders have been shared.
This feature allows proactive notification of important sharing events which facilitates collaboration, when project teams are working under tight deadlines.

With all these features [16], ownCloud ${ }^{\odot}$ can meet the requirements of organizations to store confidential data within their premises.

\section{HP PUBLIC CLOUD ${ }^{\circledR}$}

Public cloud is an open storage system where cloud users could access it either freely or pay-per-use method via Internet. The Cloud users need to pay only for the usage per hour. Hence, it could easily satisfy the On Demand Self services which is represented as a critical characteristics of Cloud computing. Examples of Public cloud include Amazon's EC2, HP Public Cloud ${ }^{\circledR}$, IBM's Blue Cloud, Microsoft's Azure and Google App Engine. Public cloud provides solutions to enterprises, industries, startups, and standalone cloud users the facility to make use of unlimited scalable computing resources with huge volume of storage capacity. Nevertheless whether it is public or private cloud, the basic concept is to promote virtualization.

HP Public Cloud ${ }^{\circledR}$ is a leading cloud computing service provider by Hewlett-Packard (HP) that pursues the idea of Converged infrastructure. It is an integration of storage, computation, networking and mobile technologies into the pools of shared self-provisioning resources [7]. The goal of HP cloud is to provide public cloud solutions for costeffective data storage and platform, elastic management with lesser administration support and more to the organizations. HP Public Cloud ${ }^{\circledR}$ handles all storage and computing needs of any organization based on OpenStack technology [9]. The architecture of HP Public Cloud ${ }^{\circledR}$ is shown in Fig. 3.

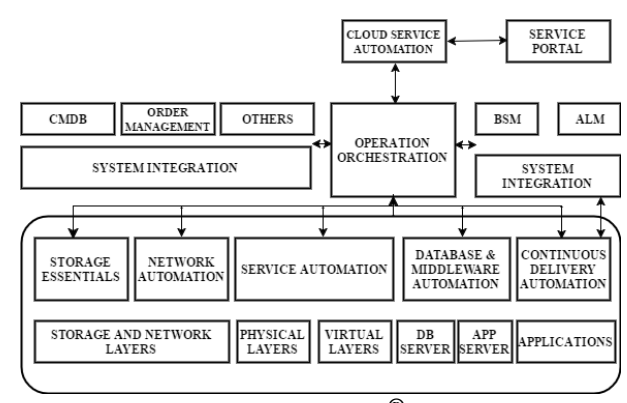

Fig. 3. HP Public Cloud ${ }^{\circledR}$ Architecture

The factors [11] that determine the use of HP Public Cloud $^{\circledR}$ technology are:

Hybrid delivery: HP Public Cloud ${ }^{\circledR}$ is a standardized hybrid delivery model where it could deliver both conventional IT services such as storage, hardware / software resources and other private cloud resources based on the accessibility of third party providers. Both corporate and non-corporate resources could be deployed. As HP Cloud provides substantial flexibility in delivering optimal mixture of different services, it is perfect for middle level organizations where there is scope for further expansion and development. 
Enterprise-grade: HP Public Cloud $^{\circledR}$ is providing the option for working from home in desktop systems as well as during travelling. The Simplicity and ease of access is well suited for any kind of business firms. The feature of multitenancy and OpenStack offers high level of scalability and sensible elasticity that fits for any grade and level of enterprise. HP Public Cloud $^{\circledR}$ is exceptionally supported by SMEs as they improve business efficiency through a full range of provisioning and self-supporting tools. In addition they help reduce operational costs due to the usage of virtual hardware and software.

Open source and transparent: The emerging issue related to cloud vendor 'lock-in' cause any enterprise to ignore a Public cloud solution. But HP Public Cloud $^{\circledR}$ is maintaining the Open Source Architecture which enables portability that led to migrating cloud to mobile apps and removes vendor 'lock-in' issues. The integration of OpenStack in HP Public Cloud ${ }^{\circledR}$ provides transparency into design infrastructure.

HP and OpenStack technology: OpenStack is free software available as open source for cloud technology which reduces the burden of purchasing licensed lock-in software by Cloud users. It is just deployed as an Operating system which can control bulk storage and large networking resources right through a data center and has created a huge user friendly eco-system for various cloud service providers. The cloud users shall choose the programming languages, software / hardware tools and frameworks from the massive pools of computing resources. Hence it is provisioned to provide Infrastructure as a Service (IaaS).

According to cloud history, HP Public Cloud ${ }^{\circledR}$ integrated with OpenStack made the unveiled change in Cloud technology to move to the elasticity features and support for infrastructure. Some of the features of HP Public Cloud ${ }^{\circledR}$ are as follows [10]:

- Accessible via APIs for developing cloud applications by cloud users and administrators

- Larger standard flavors

- High memory flavors

- Ideal for network-attached storage (NAS) architecture and provides independent fully distributed platform

- Fault tolerant, stores and retrieves arbitrary data objects

- Secure deployment

- Configuration and Server Administration managed by cloud users

- Provides API for cloud users to software defined networks

- Facilitates stable management and creation of block storage to running instances

- Offer authority and conformity for dynamic infrastructures

\section{COMPARISON OF OWNCLOUD ${ }^{\odot}$ HP PUBLIC CLOUD $^{\circledR}$}

The better choice for ownCloud ${ }^{\circledR}$ or HP Public Cloud ${ }^{\circledR}$ depends upon the project and SaaS of an organization. The focus is to give an overview about the application capabilities and not to consider on hosting / enabling the cloud. Here is a comparison of the features of private and public cloud [12]. This comparative study helps the organization moving to the cloud to decide what type of cloud environment will satisfy their needs. In this paper, the comparative parameters are studied and provided to the closest specific features that are related to both ownCloud ${ }^{\circledR}$ and HP Public Cloud ${ }^{\circledR}$.

Security: The intrinsic level of security offered by HP Public Cloud $^{\circledR}$ is always having its high standard, but the question is whether it is consistent to any specific organization or not. The major threat lies in identifying the security requirements which is very difficult to assess. As each industry sector associated with Public cloud may vary in their requirements, the cloud service provider has to set different parameters for providing the service for each organization. Hence, it is well known that Private cloud poses low security risks than Public Cloud and is considered to be less prominence because the data resides within the organization in Private cloud whereas with the Public cloud data resides in the vendors' server and database.

Cost: While Public clouds are cost effective, moderately the Private cloud would cost a significant amount that are high enough. If multi-tenancy exists, then Public cloud could offer an acceptable level of security at a lower cost when compared with Private cloud.

Data Storage: Only homogeneous data set specific to any organization can be stored in the Private cloud in huge amount. In Public cloud, any type of data of any kind of organization could be stored based on the pay-per-usage method for extensive period.

Scalability: In terms of performance, Private clouds are less scalable as it is limited to fixed number of computer systems. Any further addition of physical server or other devices should be installed and reconfigured which is a time consuming task. Whereas in Public clouds, there are many servers already installed and are used as resources when needed [13].

Software Applications: The preferable choice for any enterprise is Private cloud where they could deploy their own software applications in it. Enterprise Resource Planning (ERP) or Customer Relationship Management (CRM) applications on any enterprise are well suitable for Private cloud environment where huge set of data have to be stored securely.

Virtualization: As many companies are now interested to turn up their servers using Private cloud, they are not having adequate knowledge in Virtualization. It tends to be a component of cloud service model and enables actual cloud services for the cloud users deploying them [18].

Cloud Lock-in: Presently, there is a major concern cloud vendor lock-in where any enterprise fails to notice while using Public clouds. As Public clouds are developed and provided with licensed APIs, it is very difficult to integrate or to migrate with any other cloud platform. This may lead to any commercial users to move on to the Private cloud for better access [19]. 


\section{RESULTS AND DISCUSSION}

\section{A. Performance measures in ownCloud ${ }^{\odot}$}

It is required to measure the performance of ownCloud ${ }^{\circledR}$ which shall be considered as an important factor for enhancing the speed of cloud operations. As many database applications reside on ownCloud $^{\odot}$, the scalability of big clustered servers that are running parallel with central database storage is critical. This happens due to the database queries that are raised frequently and is difficult to scale them. The execution time of a huge set of Lines of Code (LoC) is the major consideration for measuring the performance of the type of cloud that is used in any enterprise.

As ownCloud ${ }^{\oplus}$ supports PHP programming language, it is better to take into account to test the PHP code. A flow graph has been drawn to analyze the performance metrics of ownCloud $^{\odot}$ which was generated using Blackfire tool. A test script was written to show the time difference in executing the code:

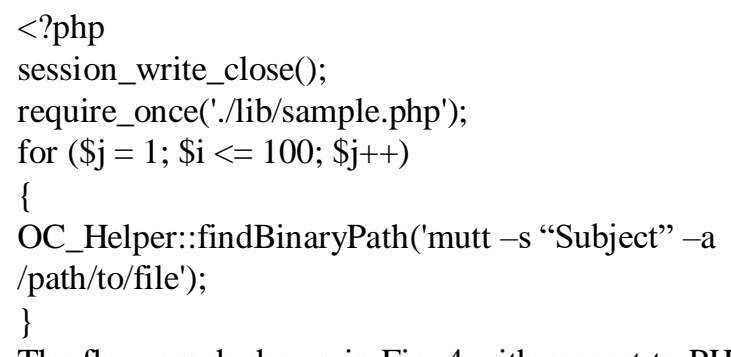

The flow graph shown in Fig. 4 with respect to PHP code running on ownCloud is supposed to be completed within two seconds with the CPU of 100 percent full load and the performance should not be affected severely on the server. It is noted that the search with the huge users' files will take more time to complete the process or else it would never end during the launching of such files in the CPU of the ownCloud server.

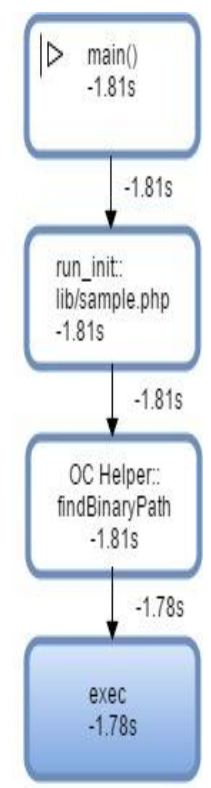

Fig. 4. PHP code performance FlowGraph in ownCloud

\section{B. Performance Considerations in HP Public Cloud ${ }^{\circledR}$}

HP Cloud supports PHP scripts where the files were stored in it and configured using public and private object storage containers. It is noted that using stream wrappers, PHP codes shall be read from and write to object storage within few minutes. As PHP Core is fully integrated and documented into the HP Cloud, the time taken for the process is substantially reduced and the HP Cloud-integrated PHP interface provides an enriched Object-oriented features.

HP Public Cloud performance shows that the functionality of the PHP-library bindings has full support for Content Distribution Network (CDN) service, Object Storage itself and offers the security services of Authentication and Authorization. HP Cloud also offers the MySQL which is an open source database and compatible with PHP programming interface. Due to this infrastructure, HP Cloud's source codes are portable and enhance its performance significantly.

\section{CONCLUSION}

Choosing which cloud to use purely depends on the need of organization, resources available in hand and also the size of the organization. If the objective is to focus on cloud platform rather than functional aspects of business organization, then ownCloud ${ }^{\odot}$ will be the better choice. If an organization owns several branches across the world, it is suggested that Public cloud can be used which includes low investment. If an organization is small scale and does not need more resources, Private cloud can be used but includes cost higher than Public cloud. When using Public cloud there may be negative loss and control over the data but if we are using Private cloud organizations retain control over the data. During the transfer of data in Public cloud under a multi-tenancy environment it implies high risk when compared to Private cloud. But the organizations need not limit to private or public cloud usage, where they can also go for hybrid or community cloud, which in turn also depends on the requirements of organization.

\section{REFERENCES}

1. The Wordpress website https://computinginthecloud.wordpress.com/2008/09/25/utilit y-cloud-computingflashback-to-1961-prof-john-mccarthy/, (2008).

2. Peter Mell, and Tim Grance, "The NIST definition of Cloud Computing. In: National Institute of Standards and Technology, Information Technology Laboratory," NIST Special Publication 800-145 15 ${ }^{\text {th }}$ Version, 2009.

3. Roadmap Working Group, "NIST Cloud Computing Standards Roadmap," Version 2 NIST Special Publication 500-291. U.S. Department of Commerce, 2013.

4. Sherif Sakr, Anna Liu, Daniel M. Batista, and Mohammad Alomari, "A survey of large scale data management approaches in cloud environments," IEEE Communications Surveys \& Tutorials, 13, pp. 311-336, April 2011.

5. The Webopedia website, http://www.webopedia.com/DidYouKnow/Internet/privatecloud-computing-vendors-to-consider.html/, (2016).

6. Matthias Henneberger and Achim Luhn, "Community Clouds - supporting business ecosystems with cloud computing,' Siemens IT solutions and services, 2010. 
7. The Hewlett Packard website, http://h10025.www1.hp.com/ewfrf/wc/document?cc=us\&lc=e n\&docname $=\mathrm{c} 02948489,(2016)$.

8. The Compucom website, http://www.compucom.com/managed-services/cloudtechnology-services/community-cloud, (2016).

9. The Softaculous website, http://www.softaculous.com/blog/features-that-makeownCloud $\odot$-a-better-data-management-application/, (2016).

10. The Hewlett Packard website, http://www.hpcloud.com/whyhp-cloud, (2016).

11. The Hewlett Packard website, http://www.hpcloud.com/hppublic-cloud-support-overview, (2016).

12. The Salesforce.com website, http://www.salesforcegeneral.com/salesforce-articles/publicvs-private-cloud.html, (2016).

13. The Zdnet.com website, http://www.zdnet.com/12-reasonswhy-public-clouds-are-better-than-private-clouds7000013156/, (2016).

14. The Slideshare website, http://www.slideshare.net/ownCloud@ /architecture-overview, (2016).

15. The ownCloud $^{\odot}$ website, http://doc.ownCloud.org/desktop/1.5/architecture.html, (2016).

16. The ownCloud $^{\oplus}$ website, https://forum.ownCloud $\odot$ .org/viewtopic.php?f=23\&t=16142, (2016).

17. The Techtarget.com website, http://searchcloudcomputing.techtarget.com/, (2016).

18. The Greenhousedata website, https://www.greenhousedata.com/blog/public-cloud-vsprivate-cloud-which-one-is-right-for-you, (2016).

19. The Techrepublic.com website, http://www.techrepublic.com/article/break-away-from-freecloud-storage-providers-by-using-owncloud/, (2016).

20. N. Usha,, V. Bhavana, Vinay and Hegde, "Various Techniques in Deployment of Application in Cloud - Public Cloud", IEEE -ELSEVIER, Society of Information processing, ICC 2014, pp. 393-399.

21. R. Arathi, Aiswarya Vijayakumar and A.S. Mahesh, "An Effective Load Balancing Mechanism in Cloud Computing Using Modified HBFA Along with the Preemptive Migration Technique", International Journal of Pure and Applied Mathematics, vol. 119, no. 10, 2018, pp. 467-478. 